\title{
Alterações Neurais Induzidas por Praguicidas
}

\section{Maria T. A. Batista, Humberto G. Rodrigues, Cristyene G. Benício \& Tales A. Aversi-Ferreira}

Os praguicidas são compostos utilizados na agricultura para melhora e proteção dos alimentos produzidos, encontram-se atualmente contaminando todo o planeta, e apesar de seus efeitos benéficos, trazem inúmeros problemas à saúde humana e ambiental. Como o homem encontra-se mais ao fim da cadeia alimentar ele está sujeito ao acúmulo desses resíduos em seu organismo e quanto maior o contato com os mesmos, maior a contaminação. Dentre os danos causados pelos praguicidas podemos citar efeitos genotóxicos, carcinogênicos, mutagênicos, teratogênicos, alterações imunológicas e hormonais e irritações e lesões ocular e cutâneas, além de alterações sobre o desenvolvimento e funcionamento do sistema neural.

Palavras-chave: toxicologia; praguicidas; sistema neural.

The pesticides are composts used in agriculture to better and protection of the foods produced and are contaminating all planet, and despite of benefic effects its generate many problems to human health and ambient. While the humans are in the last position on food net, they are target to accumulation of the pesticides in yours organisms, because while more contact with these substances more will be the contamination effects. The genotoxic, teratogenics, immunologic and hormonal alterations, irritation and lesion of the eyes, development alterations and neural system physiology are any example of dangerous effects of pesticides.

Keywords: toxicology; pesticides; neural system. 


\section{Introdução}

Os praguicidas são xenobióticos amplamente usados na agricultura. Apesar das inegáveis vantagens por eles trazidas ao ser humano como a redução do número de espécies de insetos causadores de doenças e o potencial aumento da produção agrícola com conseqüente aumento da margem de lucros obtida pelos agricultores uma vez que há um melhor aproveitamento das sementes e áreas plantadas, são igualmente inegáveis os danos causados por estes compostos ao ambiente e aos organismos vivos.

Segundo Holland", "os praguicidas são substâncias ou mistura de substâncias destinadas à prevenção, destruição ou controle de qualquer praga, incluindo vetores de doenças humanas e animais, espécies de plantas ou animais prejudiciais que possam causar danos ou interferir com a produção, processamento, estocagem, transporte ou comércio de alimentos, gêneros agrícolas, madeira, derivados da madeira ou nutrição animal, ou que possam ser administrados aos animais para o controle de insetos, ou outras pragas ou sobre seus corpos. O termo inclui substâncias destinadas para regular o crescimento da planta, desfolhante, dessecante ou agentes para o amadurecer o fruto ou prevenir sua queda prematura, e substâncias aplicadas aos produtos antes ou após a colheita para protegê-los da deterioração durante a estocagem e o transporte". A legislação brasileira classifica os praguicidas em classes de I a IV, desde produto altamente perigoso à pouco perigoso quanto ao seu potencial de periculosidade ambiental, baseando-se em parâmetros de bioacumulação, persistência, transporte, toxicidade em diversos organismos, potencial mutagênico, teratogênico e carcinogênico ${ }^{2}$.

As classes de praguicidas são constituídas por grande variedade de substâncias químicas com diferentes grupos funcionais, e diferentes formas de ação biológica e eliminação. Dentre as classes químicas encontradas temos organoclorados, organofosforados, carbamatos, piretróides, ditiocarbamatos, organoestânicos, dicarboximidas, bipiridilios, dinitrofenóis, entre outros ${ }^{3}$. Dentro destas classes, cerca de 600 ingredientes ativos estão disponíveis para a formulação dos praguicidas ${ }^{4}$.

Cada ingrediente ativo tem seu meio particular de atuação sobre seu alvo biológico, além de seus efeitos prejudiciais sobre outros organismos e sobre o ambiente. Além disso, acredita-se que muitas vezes as formulações dessas substâncias são feitas pela combinação de vários ingredientes ativos ${ }^{5}$. A agricultura constitui-se numa das principais formas de introdução dos praguicidas no ambiente. Alguns tipos como os clorados, possuem a capacidade de se conservarem ativos e manterem-se inalterados por longo período de tempo no solo, água e alimentos, aumentando os danos ambientais ${ }^{6}$.

Alguns praguicidas podem ser encontrados no solo até 20 anos após sua aplicação. Pela lixiviação, podem ser levados para os rios, que os recebem também através de efluentes industriais, esgotos e sedimentos. Uma vez iniciada a contaminação de águas, mesmo após cessar a aplicação do praguicida, as águas e os peixes que se alimentam de materiais encontrados no fundo dessas águas encontram-se ainda contaminados ${ }^{7}$. Águas superficiais podem ser contaminadas pelo transporte de praguicidas pelo ar atmosférico, ainda que durante e após a aplicação ${ }^{8}$, e mesmo que parte do produto se volatilize, este pode ainda se infiltrar no solo sendo encontrados em águas subterrâneas e em águas de poços que são utilizadas para uso doméstico e para dessedentação de animais ${ }^{9,10}$.

Os níveis de organoclorados nas águas dos oceanos tem causado o fracasso da reprodução da truta-do-mar e da águia marinha no Báltico ${ }^{11}$. Golfinhos contaminados com DDT podem ser encontrados desde o litoral paulista até regiões da Antártica ${ }^{12}$. Em vários organismos terrestres como pássaros e mamíferos, e em organismos aquáticos, tem sido detectadas concentrações tóxicas de praguicidas. Eles têm sido encontrados em plantas, invertebrados marinhos, e em pingüins da Antártida onde eles não são empregados. Resíduos de organoclorados têm se tornado parte intrínseca dos ciclos biológicos, geológicos e químicos da terra ${ }^{13,14}$.A presença de resíduos contaminantes em produtos alimentícios derivados de animais tais como carne, leite e ovos, pode ocorrer como conseqüência da aplicação direta de praguicida no animal ou quando estes ingerem alimentos (pastagens, forragens e rações) que os contenham.

A presença destes resíduos em concentrações acima das consideradas seguras pode gerar riscos toxicológicos ao homem, que se encontra mais ao fim da cadeia alimentar ${ }^{15,16}$. Em estudos feitos por Carvalho et al. ${ }^{17} \mathrm{em}$ que se analisou os níveis de resíduos organoclorados em 
produtos cárneos sob inspeção federal, foram encontrados resíduos de praguicidas em cerca de $97 \%$ das amostras de carne analisadas, tendo sido ou não sido processadas. Resíduos de praguicidas também foram encontrados em salsichas comercializadas na cidade de Santa Maria (RS), Brasil $^{18}$.

Um segundo foco de resíduos organoclorados é o leite, que constitui-se numa fonte de excreção dos mesmos pelos animais ${ }^{19}$. Sendo o leite matéria prima para vários outros produtos, encontramos resíduos de praguicidas em seus derivados, principalmente naqueles com maior porcentagem de lipídios, como o queijo. Numa pesquisa realizada por Santos et al. ${ }^{20}$ no estado do Rio Grande do Sul foi verificada a contaminação de $100 \%$ das amostras de queijo estudadas, por resíduos de organoclorados. A ingestão crônica de resíduos de praguicidas, pelo homem, potencializa efeitos tóxicos destes produtos sobre o organismo que podem ser carcinogênicos, mutagênicos, neurotóxicos e teratogênicos, além de gerarem riscos de alterações imunológicas e hormonais, irritações e lesões ocular e cutânea ${ }^{21}$.

Segundo estudos feitos por Tanabe et $a .^{22}$ os mamíferos marinhos por concentrarem organoclorados cronicamente em grande quantidade, estão entre os organismos mais vulneráveis à toxicidade. A fêmea desses animais transfere aos seus filhotes parte desses compostos presentes em seu organismo durante a gestação e a lactação ${ }^{23}$, que atravessam a barreira placentária, uma vez que os organoclorados são facilmente solúveis em lipídios. Dentre os efeitos danosos causados ao ambiente por estes produtos estão a perda de espécies de insetos ecologicamente importantes, além da seleção de outras que se tornam resistentes e o aparecimento de novas pragas, o que acaba por levar à busca por produtos mais seletivos $^{24}$.

Nos países subdesenvolvidos o uso de equipamentos de proteção é pouco freqüente, aumentando grandemente a ocorrência de intoxicações ${ }^{25}$. A constante presença destes compostos no ambiente atinge também indivíduos que não estejam envolvidos na sua produção ou aplicação. A exposição ambiental a praguicidas organoclorados tem sido associada a problemas reprodutivos em animais $^{26,27,28}$.

A contaminação da água, solos e alimentos por praguicidas é fato preocupante, mas os impactos causados por essas substâncias podem ter também uma origem ocupacional, em se tratando dos aplicadores destes produtos. Além de refletirem em vastos danos ambientais em todos os nichos ecológicos, os problemas de intoxicação refletem na saúde pública. A ingestão gradativa desses produtos presentes em água e alimentos pode ser acompanhada pela intoxicação por via respiratória ou mesmo pelo contato direto desses produtos com o tegumento ${ }^{29}$.

Os riscos para a saúde inerentes ao uso de praguicidas são maiores quanto maior a intensidade de exposição aos mesmos, que é aumentado durante o período de sua preparação e aplicação. Em um estudo realizado na Índia, efeitos genotóxicos foram caracterizados por testes de aberrações cromossômicas em linfócitos e contagem do número de micronúcleos em células retiradas do epitélio bucal de pessoas empregadas na produção de praguicidas. Quando estas pessoas foram comparadas a outras que não sofreram a mesma exposição ocupacional foi observado um aumento significante nos parâmetros analisados. Em conseqüência essas pessoas podem ter maior risco de desenvolvimento de câncer, entre outros danos.

Em estudos feitos com ratos tratados oralmente com concentrações baixas e altas dos piretróides, foram constatados mudanças nas propriedades físico-químicas da bicamada dos eritrócitos e modificações na atividade de enzimas antioxidantes ${ }^{30}$.

Parte das crianças concebidas atualmente está exposta aos praguicidas desde sua concepção, gestação e lactação, uma vez que resíduos de várias classes de praguicidas foram encontradas em amostras de sêmem de fazendeiros e também no fluído folicular e fluído amniótico de mulheres ${ }^{31}$. As crianças estão mais expostas aos praguicidas por causa da alimentação, pois comem mais por unidade de peso corporal que adultos. Além disso, rastejam por um longo período de tempo e estão constantemente levando as mãos à boca, tornando-se alvos fáceis à contaminação. Ainda que inúmeros estudos tenham sido feitos demonstrando os prejuízos causados pelos praguicidas ao ambiente e ao homem, os mesmos continuam a ser usados, algumas vezes de forma irregular, exacerbando seus efeitos tóxicos.

Danos ao sistema neural, cardíaco, endócrino e reprodutor, assim como aumento dos riscos de ocorrência de câncer podem ser causados pela presença de praguicidas 
organoclorados em água potável ${ }^{29}$.

A estrutura mais complexa do organismo é o sistema neural. Modificações na estrutura do tecido neural pode gerar comprometimentos graves para os neonatos e perturbar sua existência, além de comprometer o final da vida com demências de etiologia difícil como a doença de Alzheimer.

Alterações na intrincada rede formada pelos neurônios poderá causar sérios problemas aos indivíduos, pois defeitos neurais conduzem o indivíduo a uma vida de clausura social, diferentemente de outras etiologias. Dos principais efeitos dos praguicidas sobre a saúde de homens e animais, o comprometimento das delicadas estruturas neurais pode ser a causa de diversas patologias, algumas justificadas como o Parkinsionismo, mas outras cujas requerem estudos mais pormenorizados.

\section{Material e Métodos}

Foram realizadas buscas nas bases eletrônicas de dados: MEDLINE, Scielo, PubMed, LILACS, Periódicos CAPES e Google. Esse último se mostrou bastante eficiente, pois artigos indexados em indexadores menos conhecidos podem ser encontrados, usando-se o termo "pdf" após a digitação dos descritores. Os descritores usados foram: pesticide, toxicity.

\section{CRITÉRIOS DE INCLUSÃO}

Artigos indexados, publicados basicamente nos últimos quinze anos; artigos indexados clássicos mais antigos, livros e textos clássicos sobre os praguicidas e seus efeitos danosos sobre o ambiente e sobre os organismos vivos e sistema neural, sem considerar a data de publicação dos mesmos.

\section{CRITÉRIOS DE EXCLUSÃO}

Artigos que não abordaram as idéias principais a serem expostas no artigo.

\section{Resultados}

Os dados gerais dos artigos e textos usados neste trabalho estão listados abaixo. Alguns textos estão repetidos nos itens discriminados abaixo por conterem mais de uma abordagem geral.
Tabela 1: Dados gerais sobre os assuntos abordados nos textos utilizados na revisão

\begin{tabular}{l|c}
\hline Assunto do geral dos textos e artigos & Quantidade de artigos \\
\hline Caracterização dos praguicidas & 6 \\
Contaminação ambiental & 10 \\
Presença de residuo s em alimentos & 6 \\
Efeitos gerais da toxicidade sobre animais & 10 \\
Toxicidade neural & 21 \\
\hline Total & $\mathbf{5 3}$ \\
\hline
\end{tabular}

\section{Discussão}

Devido a alta diferenciação tecidual e celular exigidas durante a formação das estruturas componentes do sistema neural, a interferência de influências ambientais, principalmente as que causam toxicidade podem desestruturar a morfologia e fisiologia deste sistema ${ }^{32 ; 33,}$ 34;35. A maturação do sistema neural requer uma seqüência mais complexa de processos que qualquer outra estrutura do corpo, deixando este sistema mais vulnerável às influências ambientais ${ }^{36}$.

A perfeita construção do sistema neural é crucial ao funcionamento de todo o organismo ao qual pertence, uma vez que todo ele é coordenado pelo cérebro. Apesar de toda a sua importância, este sistema demonstra-se suscetível a várias substâncias tóxicas ${ }^{5,32,37,38,39}$, tanto durante seu desenvolvimento, o que pode gerar diversas neuropatias, quanto após seu amadurecimento, pois segundo estudos feitos por Tanabe et al..$^{22}$ os mamíferos marinhos por concentrarem organoclorados cronicamente em grande quantidade, estão entre os organismos mais vulneráveis à toxicidade, e a fêmea desses animais transfere aos seus filhotes parte desses compostos presentes em seu organismo durante a gestação e a lactação $^{23}$ que atravessam a barreira placentária, uma vez que os organoclorados são facilmente solúveis em lipídios, desse modo a ação desses compostos sobre o tecido neural é óbvia.

As propriedades químicas de certas substâncias tóxicas determinam seu acesso aos tecidos cerebrais. Íons com cargas iguais às do cálcio são conduzidos até aos neurônios e glia atravessando a barreira sanguínea 
cerebral com relativa facilidade ${ }^{40}$, e algumas classes de praguicidas também acessam tecidos cerebrais em virtude de sua solubilidade em lipídeos ${ }^{41}$. Comprovadamente os praguicidas são fatores que geram alterações sobre o desenvolvimento e funcionamento do sistema neural ${ }^{5}$.

Tal afirmação encontra subsídios nos fatos, já bem constatados que os efeitos de alguns praguicidas como os organofosforados têm ação contínua sobre os tecidos mesmo depois da ação imediata, isso indica permanência latente no organismo, no caso nas células adiposas por permitir a solubilidade desses compostos, no entanto, os neurônios são ricos em mielina, que pode ser um reservatório potencial dessas substâncias.

Inúmeros praguicidas interferem em várias fases do desenvolvimento do sistema neural. Algumas classes atuam principalmente durante o desenvolvimento prénatal do cérebro ${ }^{37}$. Estas interferências podem ser tanto sobre o neurônio e sua fisiologia como sobre as células da glia, que acabam por interferir na migração dos neurônios e indiretamente geram impactos sobre a construção e funcionamento do cérebro ${ }^{5}$. Durante a formação das camadas corticais, eventos tempo-espaço dependentes devem estar integrados e intimamente relacionados, e drogas e substâncias tóxicas como o álcool podem gerar ectopia e heterotopia nodular e/ou difusa nas estruturas a serem formadas, segundo dados nossos ${ }^{32 ; 33,34 ; 35}$, pois substâncias tóxicas com baixo peso molecular, afinidade lipídica, apolaridade e baixa capacidade de ligação à proteínas atravessam a placenta com facilidade ${ }^{42}$, tendo acesso ao cérebro em desenvolvimento, um dos órgãos do corpo humano mais sensíveis aos danos causados pelas substâncias tóxicas ambientais ${ }^{41}$.

A barreira sanguínea imatura de feto e crianças jovens é mais permeável a xenobióticos. Além disso o feto não tem a mesma capacidade metabólica destoxificante encontrada nos indivíduos pós natais ${ }^{43}$.

Hidrocarbonetos clorados, por exemplo, podem causar danos ao sistema nervoso central e periférico, aumento dos níveis de enzimas hepáticas e perturbações na biossíntese de esteróides ${ }^{15}$.

Em um estudo feito com ratos ficou comprovado que a exposição ao ácido 2,4-diclorofenoxiacético, um praguicida que interfere na mielinização do cérebro ${ }^{44}$, pode gerar nos filhotes mudanças no comportamento incluindo apatia, redução da interação social, movimentos repetitivos, tremores e imobilidade ${ }^{39}$. O herbicida 2,4diclorofenoxiacético é um ácido orgânico, com pKa 2,6, e possui uma solubilidade de $45 \mathrm{~g} / \mathrm{L}$ em água, é cancerígeno acarretando danos ao fígado e ao coração, ataca o sistema neural central, provocando convulsões. Sua dose letal (DL50) oral é de $370 \mathrm{mg} / \mathrm{kg}$ (em coelhos) e por via derme é de $1400 \mathrm{mg} / \mathrm{kg}$ (em camundongos) ${ }^{45}$.

Os praguicidas organofosforados influenciam o desenvolvimento do sistema neural através da inibição da acetilcolinesterase, que apresenta fundamental importância para o crescimento e diferenciação neuronal 46. Em um estudo em que se analisou a neurotoxicidade aguda do praguicida triazophos(organofosforado)emratos jovens e adultos, ficou claramente comprovada a maior sensibilidade à toxicidade apresentada pelos indivíduos jovens, refletidas nas diferenças das doses máximas por eles suportadas e nas alterações comportamentais por eles exibidas em comparação aos indivíduos adultos

A exposição intrauterina a baixos níveis de praguicidas organofosforados e PCBs tem sido associada a baixo peso corporal, maturidade gestacional e redução da circunferência da cabeça em alguns estudos ${ }^{47 ; 48}$, portanto, com efeito sobre o encéfalo, gerando preocupação acentuada com as mulheres grávidas nas zonas rurais.

Químicos ambientais que exerçam efeito excitatório sobre neurônios, gerando despolarização prolongada, são potenciais neurotóxicos do desenvolvimento, como os praguicidas organofosforados e carbamatos ${ }^{49}$.

Alguns dos praguicidas mais amplamente usados e de grande persistência ambiental atuam bloqueando canais de cloro, bloqueando receptores do ácido gamaaminobutírico (GABA) no sistema nervoso central de mamíferos, gerando hiperexcitabilidade, tremores e convulsões, dependendo do nível de exposição ${ }^{50}$, pois o GABA, juntamente com a glicina, são os principais neurotransmissores inibitórios do sistema neural central. Um dos mais utilizados praguicidas é uma glicina substituída que pode, por ação mimética, atuar nos processos inibitórios do sistema neural, pois a ação de uma substância sobre os tecidos e receptores tem sua toxicidade associada com a concentração. Talvez a ação da glicina seja maior sobre o sistema neural periférico, pois nenhum aminoácido atravessa a barreira hematoencefálica, mas potenciais ações sobre os gânglios simpáticos e parassimpáticos sacrais não devem ser 
descartadas.

Como os praguicidas são atualmente encontrados contaminando todo o planeta, trazem inúmeros problemas de saúde ao homem e ameaçam a sobrevivência das várias espécies animais e vegetais ${ }^{51}$, e estão presentes como contaminantes em produtos alimentícios derivados de animais tais como carne, leite e ovos, pode ocorrer como conseqüência da aplicação direta de praguicida no animal ou quando estes ingerem alimentos (pastagens, forragens e rações) que os contenham, a presença destes resíduos em concentrações acima das consideradas seguras pode gerar riscos toxicológicos ao homem, que se encontra mais ao fim da cadeia alimentar ${ }^{15,16}$, e considerando os dados aqui expostos como a atividade tóxica descontrolada e a alta permanência dessas substâncias no ambiente, os efeitos desses compostos são potencializados sem que ocorra um modo eficiente de controle. Alguns dados derivados de nossos estudos indicam que as glicinas substituídas exercem efeitos tóxicos sobre a membrana de eritrócitos humanos e de ratos dentro das doses recomendadas para uso na agricultura pelo fabricante ${ }^{52}$.

Dados indicam que os organoclorados estão associados ao Parkinsonismo, pois estes produtos são encontrados em maior quantidade nos cérebros de pacientes que apresentam esse tipo de demência, estando esse composto associado á degenerescência de neurônios ${ }^{53}$.

Os efeitos dos praguicidas sobre o ambiente associados com sua permanência exacerbada no meio, indica que cada vez mais pessoas poderão sofrer sua ação deletéria. Esta ação sobre sistemas orgânicos complexos, tal como o sistema neural, cujas células perderam a capacidade mitótica devido à sua alta especialização, e que, com o passar do tempo acumula substâncias tóxicas em seu citoplasma, pode ser a causa de algumas demências associadas ao envelhecimento como as síndromes associadas ao Parkinsonismo e à doença de Alzheimer.

Como a população mundial e brasileira tem aumentado sua expectativa de vida, maior número de pessoas estarão fadadas a sofrerem os danos causados pelo envelhecimento, como as demências e outras doenças degenerativas, e as ações tóxicas das várias substâncias no qual o indivíduo entra em contato durante sua vida, poderão aumentar o quadro de senescência, e os praguicidas são um desses agentes danosos, pois a maior parte dos efeitos de substâncias sobre o sistema neural está associado aos moradores de zonas rurais, cujo contato com os praguicidas é maior.

Desse modo a relação direta dos praguicidas com os problemas de saúde humana, como a geração de demência e outros problemas de saúde em geral, indica que a intoxicação por essas substâncias é um problema de saúde pública, e se considerar a permanência dessas substâncias no ambiente durante muito tempo, cada vez mais pessoas estarão sujeitas aos efeitos tóxicos dos praguicidas.

\section{Conclusões}

Os vários dados sobre os efeitos dos praguicidas sobre a saúde de animais e homens, associado com sua permanência por vários anos no meio, indica que muitas patologias estão associadas à ingestão constante dessas substâncias, e, devido à alta vulnerabilidade e importância crucial na sobrevivência e comportamento social, agressões ao sistema neural de um modo geral em fetos e neonatos de um lado e idosos do outro, podem ter como origem os efeitos deletérios dos praguicidas.

\section{BIBLIOGRAFIA}

1. Holland, P.T. Pure Appl. Chem. 1996. Chemistry And The Environment Division. Ed. IUPAC, 68, 1167.

2. http://www.andef.com.br/legislação/port84.htm, acessada em 25/02/2006.

3. http:www.inca.gov.br/inca/Arquivos/publicações/ vigilanciadocancerocupacional.pdf, acessado em 01/02/2006.

4. Richardson, M. Water Sci. Tecnol. 1998, 37, 19.

5. Colborn, T. A. Environ. Health Perspect 2006, 114, 10.

6. Bastos, L. H. 1999. Dissertação (Mestrado) - Escola Nacional de Saúde Pública da Fundação Oswaldo Cruz, Rio de Janeiro, 175.

7. Branco, S. M. O Meio Ambiente em Debate, Ed. Moderna: São Paulo, 1988.

8. Van den Berg F.; Kubiak R.; Benjey W.G.; Majewski M.S.; Yates S.R.; Reeves G.L.; Smelt J.H.; van der 
Linden A.M.A. Water Air Soil Pollut. 1999, 115, 195.

9. Rigitano, R. L. O.; Barbosa, T. M. L. Pesqui. Agropecu. Bras. 1994, 29, 955.

10. Moreira, L. F.; Cruz, J. C. S. Viçosa: Departamento Técnico Emater, 1996.

11. http://www.topos.com.br/cordella/prag.htm.,1999, acessada em 25/02/2006.

12. Yogui, G. T. 2002. Dissertação (Mestrado) Instituto Oceanográfico, Universidade de São Paulo, São Paulo, 134.

13. EPA - U. S. Environmental Protection Agency. Pesticide Monitoring, Phase II Fact Sheet. Series 7 (of 14). Washington: Environmental Protection Agency, 1991.

14. Li, Y. F.; Scholtz, M. T.; Heyst, B. J. V. Environ. Sci. Technol. 2003, 37, 3493.

15. Huber, W. G., Chemical In Animal Products. In: Hubbert, W. T. et al. Diseases transmitted from animal to man. Springfield: Editora Charles C. Thomas, 1975.

16. Lara, W. T. Revista do Instituto Adolfo Lutz 1972, 32, 89.

17. Carvalho, J. P. P.; Nishikawa, A. M.; Fay, E. F. Rev Saude Publica 1980, 14, 408.

18. Junior, S. B.; Santos, J. S.; Xavier, A. A. O.; Weber, J.; Leães, F. L.; Costabeber, J.Ciência Rural 2004, 34, 1593.

19. Waliszewski, S. M.; Pardío, V. T.;Waliszewski, K. N.; Chantiri, J. N.; Aguirre, A. A.; Infanzón, R. M.; Rivera, J. Sci. Total Environ 1997, 208, 127.

20. Santos, J. S. Xavier, A. A.O.; Ries, E. F.; Costabeber, J. H.; Emanulli, T. Ciência Rural 2006, 36, 630.

21. WorldHealth Organization.Polychlorinated Biphenils and Terphenyls (PCBs and PTCs): health and safety guide. Geneva: World Health Organization, 1992.

22. Tanabe, S.; Iwata, H.; Tatsuakawa, R. Sci. Total Environ. 1994, 154, 163.

23. Aguilar, A.; Borrell, A. Arch. Environ. Contam.
Toxicol. 1994, 27, 546.

24. Senent, J. A. Poluição. Rio de Janeiro: Editora Salvat, 1979.

25. Forget, G. Int. Dev. Res.Cent. Rep. 1989, 18, .7.

26. Delong, R.; Gilmartin, W. G.; Simpson, J. G. Science 1973, 181, 1168.

27. Fry, D.M.; Toone, C.K. Science 1981, 213, 922.

28. Ware, G.W. Residue Reviews 1975. 59, 119.

29. Rand, G. M.; Petrocelli, S. R. Fundamentals of Aquatic Toxicology Methods and Aplication, Washington: Hemisphere Publishing Co, 1985.

30. Nasuti, C.; Cantalamessa, F.; Falcioni, G.; Gabbianelli, R. Toxicology 2003, 191, 233.

31. Foster, W.; Chan, S.; Platt, L.; Hughes, C. J. Clin. Endocrinol. Metab. 2000, 85, 2954.

32. Aversi-Ferreira, T. A.; Morais, J. O. R.; Ferreira, N. R.; Penha-Silva, N. Braz. J. Morphol. Sci. 2004, 21, 97.

33. Aversi-Ferreira, T.A., Penha-Silva, N. Biosci. J. 2005a, 21, 151 .

34. Aversi-Ferreira, T. A.; Corrêa, N. C. R.; Morais, J. O. R.; Penha-Silva, N. Neurociências 2005b, 2, 1.

35. Aversi-Ferreira, T. A.; Rodrigues, H. G.; Neres, A.C.; Fonseca, L.C.; Penha-Silva, N. Biosci. J. 2006, 22, 99.

36. Rodier P. M. Environ. Health Perspect 1994, 102, 121.

37. Qiao, D.; Seidler, F. J.; Abreu-Villaça, Y.; Tate, C. A.; Cousin, M. M.; Slotkin, T. A. Dev. Brain Res. 2004, 148, 43.

38. Eriksson, P.; Talts, V. Neurotoxicology 2000, 21, 37.

39. Bortolozzi, A. A.; Duffard, R. O.; Evangelista De Duffard, A. M. Neurotoxicol Teratol. 1999, .21, 451.

40. Kerper, L. E. Hinkle, P. M. Toxicol. Appl. Pharmacol. 1997, 146, 127.

41. Dietrich, K. N.; Eskenazi, B.; Schantz, S.; Yolton, K.; Rauh, V. A.; Johnson, C .B.; Alkon, A.; Canfield, 
R. L.; Pessah, I. N.; Berman, R. F. Environ. Health Perspect. 2005, 113, 1437.

42. Slikker W Jr, Miller RK. 1994. In: Developmental Toxicology (Kimmel CA, Buelke-Sam J, eds). New York: Raven Press, 245-283

43. Rozman, K. K.; Klassen, C. D. Absorption, distribution, and excretion of toxicants. In: KLASSEN, C. D.; AMDUR, M.; O.; DOULL, J. Toxicology: The basic Science of Poisons. New York: Mc Graw-Hill, 1996.

44. Rosso, S. B.; Garcia, G. B.; Madariaga, M. J.; de Duffard, A. M. E.; Duffard, R. O. Neurotoxicology 2000, 21, 155.

45. Vieira, E. M. Prado, A. G. S.; Landgraf, M. D.; Rezende, M. O. O. Quim. Nova 1999. 22, 305.

46. Weiss, B.; Spyker, J.M. Pediatrics 1974, 53, 851.

47. Berkowitz, G. S.; Wetmur, J. G.; Birman-Deych, E.; Obel, J.; Lapinski, R. H.; Godbold, J. H.; Holzman, I. R.; Wolff, M.S.; Environ. Health Perspect. 2004, $112,388$.

48. Whyatt, R. M.; Rauh, V.; Barr, D. B.; Camann, D. E; Andrews, H. F.; Garfinkel, R.; Hoepner, L. A.; Diaz, D.; Dietrich, J.; Reyes, A.; Tang, D.; Kinney, P. L.; Perera, F. P. Environ. Health Perspect 2004, 112, 1125.

49. Slotkin, T. A. Environ. Health Perspect 1999, 107, 71.
50. Narahashi T.; Ginsburg, K. S.; Nagata, K.; Song J. H, Tatebayashi, H. Neurotoxicology 1998, 19, 581.

51. Flores, A. V.; Ribeiro, J. N.; Neves, N. N.; Queiroz, E. L. R. Ambiente e Sociedade 2004, 7, 111.

52. Batista, M. T. A.; Rodrigues, H. G.; Fonseca, L. C. Bonnetti, A. M.; Penha-Silva, N.; Aversi-Ferreira, T. A. 2007. in: XXX Reunião anual da sociedade brasileira de química, 2007. Anais, Águas de Lindóia. CD ROM.

53. Nunes, M. V.; Tajara, E. H. Rev Saude Publica 1999, 32,372 .

\section{Maria T. A. Batista?, Humberto G. Rodrigues', Cristyene G. Benício' \& Tales A. Aversi-Ferreira ${ }^{*}$}

${ }^{1}$ Laboratório de Bioquímica e Neurociências, Departamento de Morfologia, Instituto de Ciências Biológicas, Universidade Federal de Goiás (UFG)

*e-mail: aversiferreira@hotmail.com 